\title{
e-Phaïstos
}

e-Phaïstos

Revue d'histoire des techniques / Journal of the history

of technology

VII-2 | 2019

La sauvegarde du patrimoine scientifique

\section{La Vallée de l'Étrange de Mori Masahiro}

Importance et impact sur l'esthétique et la conception des robots

Masahiro Mori's The Uncanny Valley: Significance and Impact on Aesthetics and Robot Design

Karl F. MacDorman

\section{(2) OpenEdition}

\section{Journals}

Édition électronique

URL : http://journals.openedition.org/ephaistos/5333

DOI : 10.4000/ephaistos.5333

ISSN : 2552-0741

Éditeur

IHMC - Institut d'histoire moderne et contemporaine (UMR 8066)

Référence électronique

Karl F. MacDorman, «La Vallée de l'Étrange de Mori Masahiro », e-Phaïstos [En ligne], VII-2 | 2019, mis en ligne le 15 octobre 2019, consulté le 10 décembre 2020. URL : http://journals.openedition.org/ ephaistos/5333 ; DOI : https://doi.org/10.4000/ephaistos.5333

Ce document a été généré automatiquement le 10 décembre 2020.

Tous droits réservés 


\title{
La Vallée de l'Étrange de Mori Masahiro
}

\author{
Importance et impact sur l'esthétique et la conception des robots \\ Masahiro Mori's The Uncanny Valley: Significance and Impact on Aesthetics and \\ Robot Design
}

Karl F. MacDorman

\section{RÉFÉRENCE}

Mori, Masahiro. The Uncanny Valley Phenomenon. Energy, 7(4), Esso Standard Oil, 1970, pages 33-35.

Je souhaiterais exprimer ma reconnaissance à ceux qui m'ont gracieusement aidé à traduire en français l'essai précurseur de Mori Masahiro. Danny Pham a effectué une traduction juste de ma traduction anglaise de 2012 avec Kageki Norri comme référence, alors que je me refamiliarisais moi-même avec l'essai de Mori en japonais. Nomura Tatsuya m'a conseillé sur les passages de Mori les plus difficiles. Lucile Julaud, Emilien Kopp et Elodie Marechal ont revu et corrigé ma traduction du japonais vers le français.

\section{Préface}

1 En 1970, Mori Masahiro a écrit un essai innovant sur l'esthétique de la forme humaine ${ }^{1}$. Il s'est particulièrement préoccupé de la façon dont l'apparence humaine des robots modifie la manière dont ils sont perçus. Mori, lorsqu'il était enfant, fut fortement perturbé par une exposition de personnages en cire totalement statiques (Kageki, 2012). Il fit à nouveau l'expérience de ce sentiment étrange, bien des années plus tard, en tant que professeur d'ingénierie de contrôle à l'Université de Technologie de Tokyo, alors qu'il révisait une main myoélectrique (prothèse motorisée) qui semblait parfaitement humaine, mais n'était pourtant pas perçue comme telle. Ce sont ces 
expériences qui l'ont incité à réfléchir au futur des robots humanoïdes et ont inspiré cet essai.

2 À travers une série d'observations et d'expériences de pensée, il décrit dans son essai la corrélation entre le degré de ressemblance anthropomorphique d'un robot et le niveau de sympathie ressenti à son égard. Plus les robots seraient similaires à l'homme, plus notre sympathie pour eux augmenterait - mais seulement jusqu'à une certaine limite. En se rapprochant d'une apparence humaine parfaite, mais sans toutefois l'atteindre, notre réponse changerait soudainement et passerait de la sympathie à l'aversion. Ce changement, qui sera également le titre de son essai, Mori l'appelle bukimi no tani genshō 不気味の谷現象 - le phénomène de la vallée de l'étrange. Il le présente comme étant la manifestation d'un instinct de conservation, protégeant notre espèce de l'exposition aux cadavres et autres espèces apparentées et dont l'effet se verrait amplifié par le mouvement.

3 L'essai de Mori a d'abord paru dans Energy, un journal obscur et ayant cessé de paraitre, publié par Esso Standard Oil, la filiale japonaise d'un conglomérat américain (Mori, 1970). Puis, c'est en 1978 que Jasia Reichardt introduira le concept de la vallée de l'étrange dans le monde anglophone avec son livre intitulée Robots : Fact, Fiction and Prediction (Reichardt, 1978a). Reichardt (1978b) reçut de Sugiura Kohei, graphiste récompensé, un résumé de l'essai en anglais. En traduisant bukimi 不気味 par uncanny, elle créera malgré elle un lien entre les travaux précédent sur l'inquiétante étrangeté (das Unheimlich) de Ernst Jentsch (1906) et Sigmund Freud (1919), bien que ni l'un ni l'autre n'aient été à l'origine de l'inspiration de Mori (Jochum et Goldberg, 2015).

4 Traduire shinwakan 親和感 par familiarité par contre, a posé un plus grand problème. Shinwakan est un néologisme inventé par Mori et représente l'ordonnée sur son graphique. C'est ce qu'il définit comme étant l'expérience de la présence et de la connexion sociale. Sa polarité opposée correspond à l'étrangeté ressentie devant l'humain sans esprit ou la machine consciente (Gray et Wegner, 2012). Le traduire par familiarité est problématique, car lorsqu'une entité tombe dans la vallée de l'étrange, sa familiarité ne peut chuter en dessous de zéro, comme elle le ferait dans le graphique de Mori, tout comme sa nouveauté ne peut s'élever à plus de 100\%. En 2012, alors que nous préparions, Kageki Norri et moi, une traduction autorisée en anglais (Mori, 2012a) de l'essai, nous avons accepté la suggestion de Mori de traduire shinwakan par affinité. Mais si l'on garde en tête le sens de ressemblance ou de similitude pour le mot affinité, la compréhension du texte de Mori peut s'en trouver compromise. En effet, puisque sur son graphique l'abscisse représente la ressemblance anthropomorphique, l'axe des ordonnées ne peut contenir le même sens. Il serait donc plus clair pour le lecteur d'utiliser le terme sympathie, plutôt qu'affinité. Mes propres recherches sur le terme shinwakan ont conduit à la reconnaissance de deux dimensions différentes dans sa signification: l'absence d'étrangeté et la sympathie, ou chaleur humaine (MacDorman et Chattopadhyay, 2016; MacDorman et Entezari, 2015). En effet, la chaleur humaine est la dimension première de la perception de l'autre; elle est ensuite suivie par la compétence (Fiske, Cuddy et Glick, 2007). Toutefois, traduire ce terme seulement par sympathie ou chaleur est également contradictoire: des ennemis peuvent manquer de sympathie l'un envers l'autre, sans pour autant se percevoir étrangement. Le lecteur est donc encouragé à traiter le mot affinité comme un terme technique combinant plusieurs concepts. 
5 Malheureusement, en 2005, la traduction incomplète et faite à la hâte en compagnie de Minato Takashi pour un usage personnel (MacDorman, 2005), est celle qui a finalement été utilisée à grande échelle et a propagé des erreurs de traduction en anglais, et plus tard en français, à travers la traduction indirecte d'Isabel Yaya (Mori, 2012b). Traduire cet essai peut se révéler être un défi. Car Mori adopte un style littéraire et aborde le sujet sous différents angles, contrairement à l'approche plus communément retrouvée dans les écrits scientifiques, qui consiste en une exposition linéaire. Son style est composé d'un vocabulaire riche et varié, comprenant aussi bien des termes techniques que des expressions familières et des nuances précises qui peuvent facilement se perdre dans la traduction. Étant donné l'influence durable de l'essai de Mori, qui sera très probablement amenée à se développer encore, à mesure que la technologie devient plus humaine, le français mérite une traduction définitive. C'est ce que je me propose de faire ici.

6 Pour illustrer la vallée de l'étrange, Mori dans son essai, fait référence aux formes traditionnelles de théâtre japonais, en particulier le bunraku 文楽et le nō. Le bunraku utilise des marionnettes mesurant environ un mètre de haut, costumées de manière élaborée et généralement contrôlées par trois marionnettistes vêtus de noir (Illustration 1). Mori prend pour exemple le bunraku pour illustrer la manière dont une performance magistrale peut rendre ces marionnettes humaines et leur permettre ainsi de gagner l'affection du public. Ce sont des acteurs humains qui jouent dans les pièces de $n \bar{o}$, le protagoniste et parfois un compagnon, portent des masques afin de représenter la jeunesse, la vieillesse, les personnages féminins, mais également des personnages non humains tels que des animaux, des fantômes, des démons ou encore des divinités. Le masque okina 翁 représente un homme âgé, alors que celui du yase otoko 瘦男, ressemblant à un homme émacié, représente un fantôme de l'enfer (Illustration 2). Mori utilise les masques yase otoko et okina afin d'illustrer respectivement les côtés moins réalistes et plus réalistes de la vallée de l'étrange, bien qu'aucun de ces deux masques ne soit vraiment très réaliste. 
Illustration 1 : Marionnette de bunraku

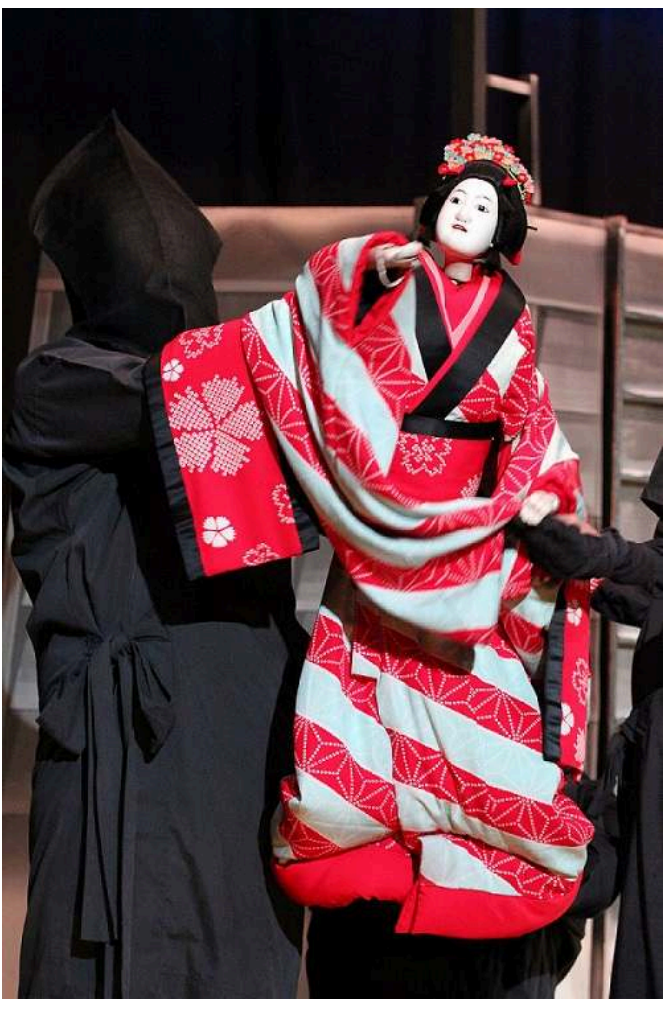

Ningyō jōruri 人形淨瑠璃, aussi appelé bunraku, est un spectacle musical de marionnettes qui provient

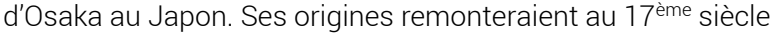

chee-Onn Leong () 123rf.com 
Illustration 2 : Les masques yase otoko et okina

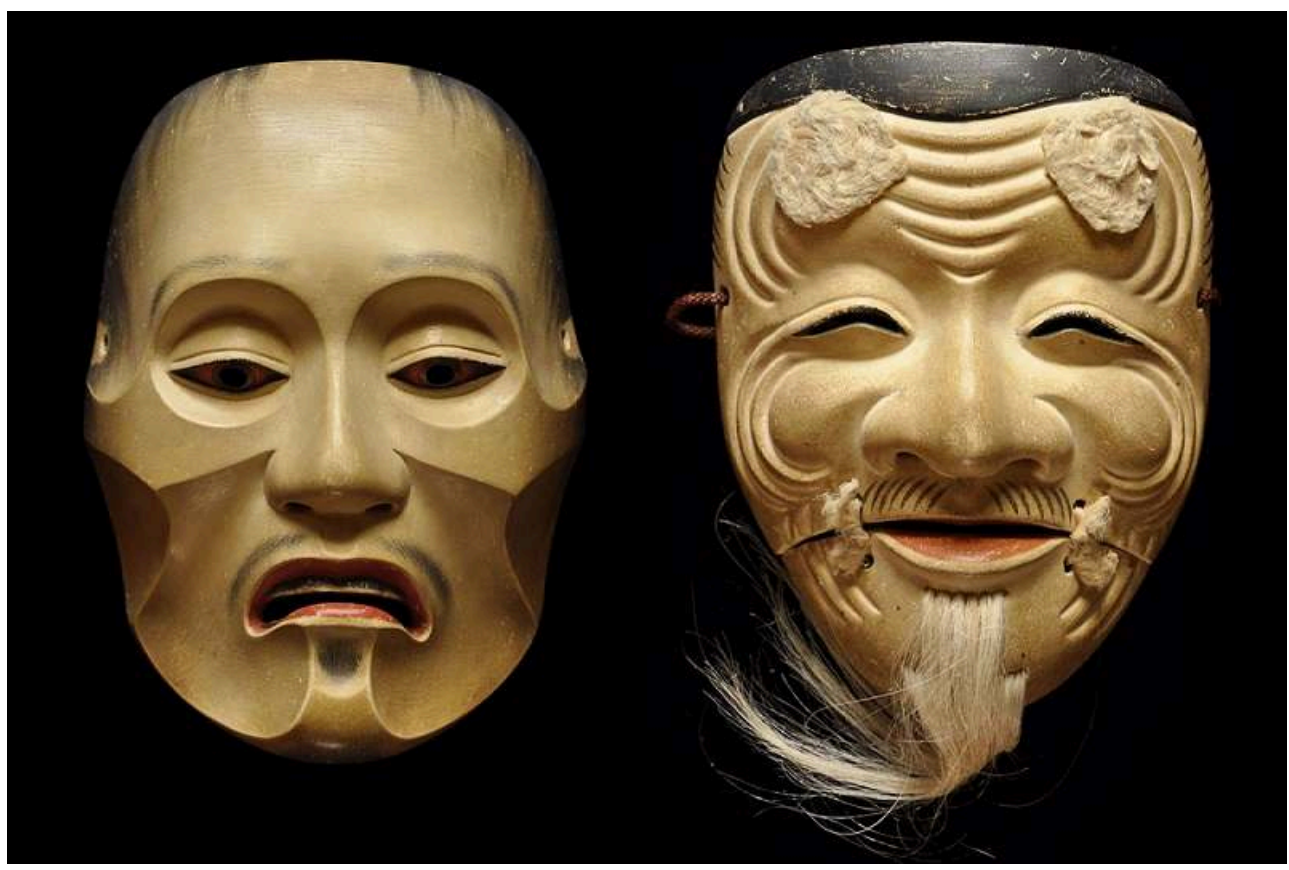

Le nō est une forme de théâtre musical japonais qui date du 14 ème siècle. Le masque yase otoko (à gauche), montrant le visage d'un homme émacié, représente un fantôme de l'enfer. Le masque okina (à droite) représente un homme âgé. Les masques ici présentés ont été taillés par Ichiyu Terai à Kyoto au Japon

Shuhei Terai (c) nohmask.jp

7 Mori ne proposa pas tant son schéma de la vallée de l'étrange comme hypothèse formelle, mais plutôt comme une ligne directrice pour les concepteurs de technologie, et c'est ainsi que ce schéma fut interprété au début. Afin de réduire le risque qu'un modèle ne paraisse trop étrange, il préféra favoriser les modèles plus abstraits pour éviter un anthropomorphisme trop important.

Pendant une trentaine d'années après la publication de son essai, l'interdiction de poursuivre un design anthropomorphique poussé initiée par Mori était devenue un principe de base parmi les communautés robotiques du Japon et au-delà. Ce qui est d'ailleurs curieux, car la culture et la religion japonaise n'avaient jusque-là jamais résisté à la mécanisation de la forme humaine (Yamamoto, 1983). Les poupées mécaniques (karakuri ningyō からくり人形) y sont populaires depuis l'époque d'Edo (1603-1867) et les robots humanoïdes sont représentés plutôt favorablement dans la culture populaire (MacDorman, Vasudevan et Ho, 2009). Néanmoins, si des androïdes perçus comme trop étranges retournaient l'opinion publique contre la robotique, cela pourrait menacer certaines des priorités nationales du gouvernement japonais, comme le développement et l'utilisation des robots pour l'accompagnement des personnes âgées par exemple. Par conséquent, c'est à cause de la vallée de l'étrange que certaines commissions de subventions ont été réticentes à attribuer des fonds aux projets visant à fabriquer des robots à fort degré de ressemblance anthropomorphique, autrement dit, des androïdes.

En 2003, j'ai rejoint le laboratoire d'Ishiguro Hiroshi, un professeur en robotique de l'Université d'Osaka, afin de travailler sur des projets concernant les andrö̈des. Ishiguro s'était associé avec une entreprise d'animatroniques basée à Tokyo, Kokoro Co. 
Ltd, pour créer «Repliee R1» en 2001, puis «Repliee Q1» en 2003. "Repliee R1», alimenté par neuf moteurs à courant continu était une copie de Lisa, 5 ans, la fille d'Ishiguro. "Repliee Q1 ", alimenté par 33 actionneurs pneumatiques, bien que de genre neutre, fut habillé en femme. Le but d'Ishiguro était d'utiliser un androïde à la place d'un humain pour pouvoir étudier l'interaction d'humain à humain. Cette approche, Ishiguro la nomma « la science androïde » et avec ces deux robots, il débuta des collaborations avec des chercheurs en psychologie du développement, ainsi qu'en d'autres domaines.

En 2004, j'ai observé que même si la vallée de l'étrange pouvait potentiellement mettre en danger l'acceptation des androïdes par le grand public, elle jouait pourtant un rôle crucial dans la conduite de la science androïde (MacDorman et Ishiguro, 2006). Si nous appliquons une théorie d'interaction humaine dans un androïde, nous pouvons alors savoir lorsqu'un androïde produit des réponses non humaines. Toutefois, si nous mettons en place une théorie d'interaction humaine dans un robot d'apparence mécanique, nous ne pourrons pas savoir à quel moment le robot produira des réponses non humaines. Un robot d'apparence mécanique n'active pas dans le cerveau de l'observateur le modèle d'un autre être humain et ne peut donc pas déroger aux prédictions de ce modèle (Saygin et al., 2012 ; Urgen et al., 2018).

11 Même après le succès de son androïde le plus récent, il fut difficile pour Ishiguro de faire subventionner ses recherches sur les androïdes à cause du l'essai de Mori. Il réussit finalement à persuader Inoue Hirochika de subventionner le projet $\mathrm{NEDO}^{2}$ afin d'actualiser « Repliee Q1 », qui passa de 33 à 42 actionneurs pneumatiques et ceci afin de permettre au robot d'avoir des expressions faciales ainsi que des mouvements au niveau des mains, en préparation de l'Exposition Spécialisée de 2005 à Aichi au Japon. Le robot fut doté d'une nouvelle tête et de nouvelles mains, conçues d'après la journaliste Fujii Ayako de la NHK et fut même en mesure de lire les informations à la télévision. Malgré tout, et selon l'accord avec Inoue, les membres du laboratoire et moimême devions être très prudents en présentant le robot au public. Il ne devait jamais être montré à l'arrêt, ni partiellement désassemblé, car son apparence aurait pu heurter l'opinion publique. La NHK avait formulé les mêmes demandes.

12 À travers le livre de Reichardt, le concept de la vallée de l'étrange aux États-Unis a été redéfini selon un principe de base totalement différent de celui utilisé au Japon. Bien qu'encore lié aux robots humanoïdes dans les cercles académiques, depuis 2001, son usage est devenu extrêmement populaire parmi les critiques et le grand public, pour décrire (la qualité de la modélisation et l'animation) des personnages humains dans les films d'animation 3D et les jeux vidéo. Cette année-là fut marquée par la sortie du film d'animation très réaliste Final Fantasy: Les Créatures de l'esprit. Bien qu'incroyable du point de vue technique, le film fit un flop au box-office. Les critiques ont souvent blâmé, et peut-être à tort, la vallée de l'étrange pour cet échec. Ces reproches ont en tout cas continué à se succéder et la suite de films produits par Robert Zemeckis en a fait les frais en commençant par Le Pôle express en 2004 et en culminant en 2011 avec le très attaqué Milo sur Mars, et la fermeture par Disney du studio du film, ImageMovers Digital. Ces films combinent des animations 3-D et de la capture de mouvements d'acteurs humains (Butler et Joschko, 2009 ; Freedman, 2012). Lorsque l'apparence et les mouvements des personnages sont mal coordonnés, il devient alors difficile pour le public de s'identifier à eux. 
13 En 2005 déjà, les journalistes et le public en général utilisaient la vallée de l'étrange comme concept fourre-tout pour désigner tout défaut dans l'animation réaliste d'un personnage. Le fait d'attribuer à la vallée de l'étrange les évaluations négatives reçues par l'animation est devenu un argument circulaire. Un argument non circulaire analyserait le phénomène en relation avec diverses variables de stimuli, d'affection et du comportement, puis chercherait ensuite à découvrir les mécanismes psychologiques et neurocognitifs sous-jacents qui sont à l'œuvre, ainsi que leurs évolutions.

Selon Mori, l'influence de son essai a vraiment explosé à partir de 2005, lorsque Christian Keysers, Frank Pollick et moi-même avons organisé un atelier sur la vallée de l'étrange (Kageki, 2014). Même si ce n'est pas tout à fait correct, cette année-là marque sûrement le début de l'investigation scientifique de la vallée de l'étrange, qui n'a fait que s'accélérer depuis. Certains chercheurs ont exploré son implication dans l'interaction homme-robot (Mathur et Reichling, 2016; Mitchell et al., 2011) et l'animation 3D (Burleigh et Schoenherr, 2014; MacDorman, 2019; MacDorman et Chattopadhyay, 2016; Seyama et Nagayama, 2007; Tinwell et al., 2011), alors que d'autres ont enquêté sur ses origines biologiques (Saygin et al., 2012 ; Urgen et al., 2018) et sociales (MacDorman et Entezari, 2015 ; Tondu et Bardou, 2011). L'hypothèse reste controversée, avec environ deux tiers des études publiées soutenant le phénomène (Kätsyri et al., 2015 ; Lay et al., 2016). Avec l'avancée de la technologie et des chercheurs concevant des robots de plus en plus anthropomorphiques, l'intérêt pour la vallée de l'étrange ne fera que s'intensifier.

\section{Mori MASAHIRO, La Vallée de l'Étrange, traduit du japonais par Karl F. MacDorman}

\section{La vallée de l'affinité}

15 Le terme mathématique "fonction monotone " décrit une relation binaire dans laquelle la valeur de la fonction $y=f(x)$ augmente continuellement en rapport avec la variable « $x$ ». Par exemple, si l'effort $x$ progresse, le bénéfice $y$ s'accroît, ou si la pression sur la pédale d'accélérateur augmente, la voiture roule plus vite. Ce genre de relation est répandu et facile à comprendre. Étant donné que de telles fonctions s'appliquent à de nombreux phénomènes de la vie quotidienne, nous pourrions croire, à tort, qu'elles recouvrent tout type de relations. On peut parfois observer des individus s'obstinant dans une seule approche face à un problème; ils s'entêtent par exemple à pousser, sans jamais envisager l'efficacité de la traction. C'est pourquoi les gens sont en général déconcertés face aux phénomènes non-représentables par ce genre de fonction.

L'ascension d'une montagne est un exemple de fonction qui n'augmente pas continuellement. La présence de collines et de vallées fait que la progression $(x)$ d'un randonneur jusqu'au sommet et l'altitude $(y)$ de ce dernier, n'augmentent pas simultanément. J'ai remarqué que plus l'apparence extérieure d'un robot se rapproche de celle d'un être humain, plus notre affinité vis-à-vis de lui augmente, mais que, dépassé un certain point, le sentiment d'affinité redescend dans une «vallée » que je nomme ici la vallée de l'étrange. 
Figure 1 : La vallée de l'étrange

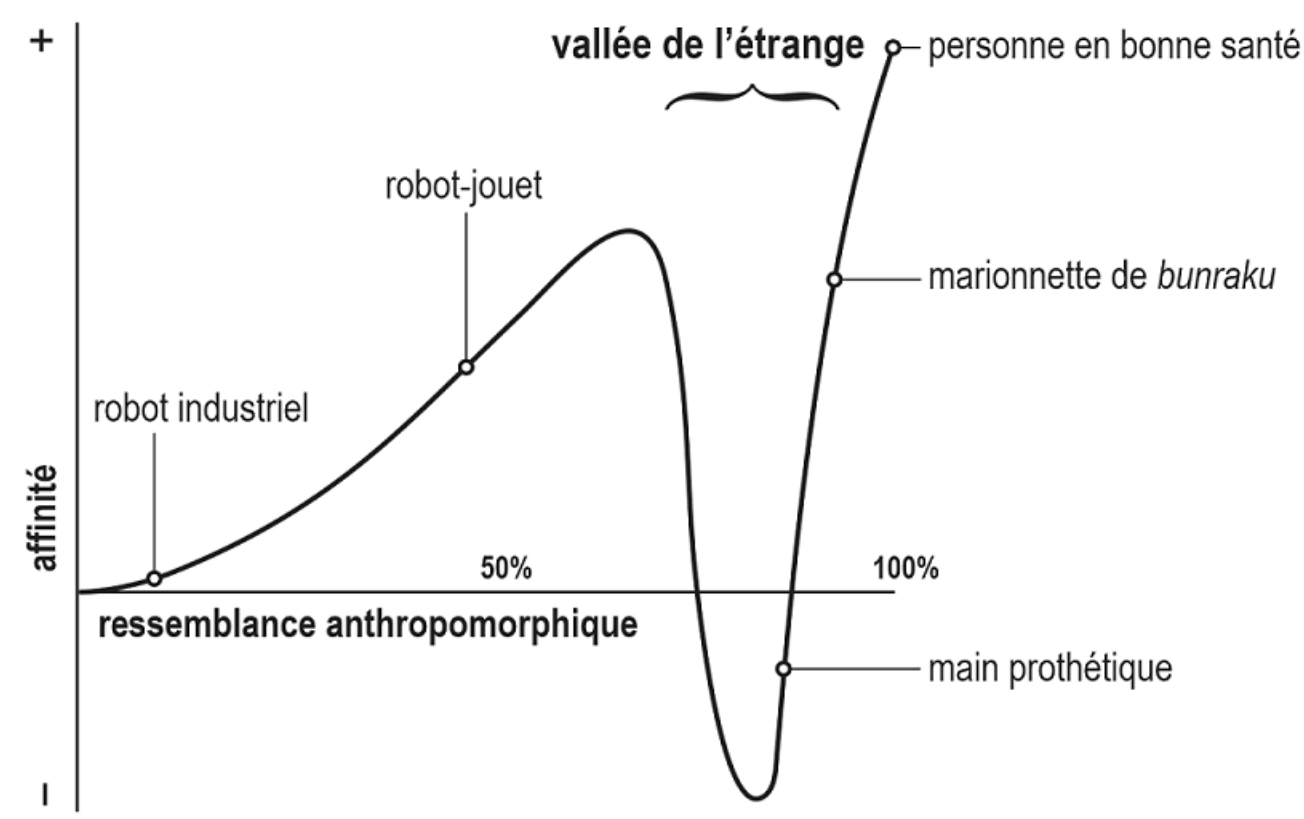

Ce graphique illustre la vallée de l'étrange, à savoir la relation entre le degré de ressemblance anthropomorphique d'une entité et le sentiment d'affinité qu'elle induit chez l'observateur.

Karl F. MacDorman

De nos jours, les robots industriels attirent l'attention puisqu'ils jouent un rôle important dans la réduction du nombre d'ouvriers dans les usines. Comme nous le savons, ces robots ne font qu'étendre, contracter et faire pivoter leurs bras. Sans visage ou sans jambes, ils ne paraissent pas humains. Ils sont conçus uniquement pour être fonctionnels. Dans ce contexte, les robots doivent effectuer des tâches similaires à celles réalisées par les ouvriers, sans pour autant devoir leur ressembler physiquement. Néanmoins, étant donné leur manque d'apparence anthropomorphique, les gens ressentent difficilement de l'affinité pour eux ${ }^{3}$. Sur un graphique montrant l'évolution de l'affinité ressentie en fonction du degré d'anthropomorphisme, le robot industriel se trouvera près de l'origine (voire fig. 1).

Cependant, les concepteurs de robot-jouet peuvent se concentrer davantage sur son apparence que sur sa fonction. Dans ce cas, bien qu'il doive essentiellement être mécanique et robuste, le robot pourra commencer à avoir une forme humanoïde, avec un visage, deux bras, deux jambes et un torse. Les enfants s'attachent en général facilement aux robots-jouets humanoïdes. Par conséquent, un tel robot se trouvera plus qu'à mi-hauteur du premier pic de la figure 1 .

Créer un humain artificiel étant un des objectifs de la robotique, de nombreux efforts se concentrent sur la fabrication de robots à apparence humaine ${ }^{4}$. Prenons l'exemple du bras d'un robot: si l'on habille la barre de fer recouverte d'écrous qui lui sert de bras d'un semblant de peau et de muscles, l'apparence change pour devenir plus humaine et le sentiment d'affinité ressenti pour la machine augmente proportionnellement.

Beaucoup parmi nos lecteurs ont déjà été confrontés à des personnes présentant un handicap moteur. Qui n'a pas également ressenti de la compassion pour ceux à qui il manque une main ou une jambe ou qui portent une prothèse? En raison des progrès récents des techniques de fabrication, nous ne pouvons pas toujours distinguer au 
premier coup d'œil une main prothétique d'une véritable main. Certains modèles simulent les rides, les veines, les ongles et même les empreintes digitales. Même si elle est similaire à une vraie main, la couleur d'une main prothétique est plus rose, comme si elle sortait d'un bain chaud.

21 Actuellement, il est indéniable que la main prothétique a atteint un niveau anthropomorphique très poussé, à l'image de ce qui se fait pour les dentiers. Néanmoins, une fois que nous nous apercevons que la main qui paraissait si réelle au premier abord est en réalité artificielle, nous sommes saisis d'inquiétude. Pouvons-nous serrer cette main? Devant sa froideur, sa poigne molle et son absence d'os, nous reculerions certainement avec effroi.

À ce moment, l'impression d'affinité disparaît et la main devient dérangeante. Mathématiquement parlant, cela peut être représenté par une valeur négative. Dans ce cas, bien que l'apparence d'une main prothétique soit assez proche de celle d'une main humaine, le niveau d'affinité ressenti est négatif et sur la figure 1, cette main se retrouve presque au fond de la vallée.

23 Je ne pense pas non plus, en regardant de près, que l'on puisse dire qu'une marionnette de bunraku ressemble à un être humain. Son réalisme en termes de taille, de grain de peau, etc., est bien loin d'atteindre celui d'une main prothétique réaliste. Mais lorsque nous assistons à un spectacle de marionnettes, nous sommes assis à une certaine distance de la scène. La taille réelle des marionnettes n'est plus perceptible, seules restent les proportions et les mouvements des mains et des yeux qui par le jeu des marionnettistes deviennent très humains. Nous sommes, en tant que spectateurs, emballés par l'esthétique créée par le spectacle, la marionnette nous apparaît ainsi sympathique et nous ressentons une grande affinité pour elle, elle sera donc représentée sur la pente supérieure de la vallée.

24 À travers tous ces exemples, nos lecteurs devraient pouvoir à présent comprendre le concept de la vallée de l'étrange. Examinons maintenant en détail la relation entre la vallée de l'étrange et le mouvement. 
Figure 2 : L'effet du mouvement sur la vallée de l'étrange

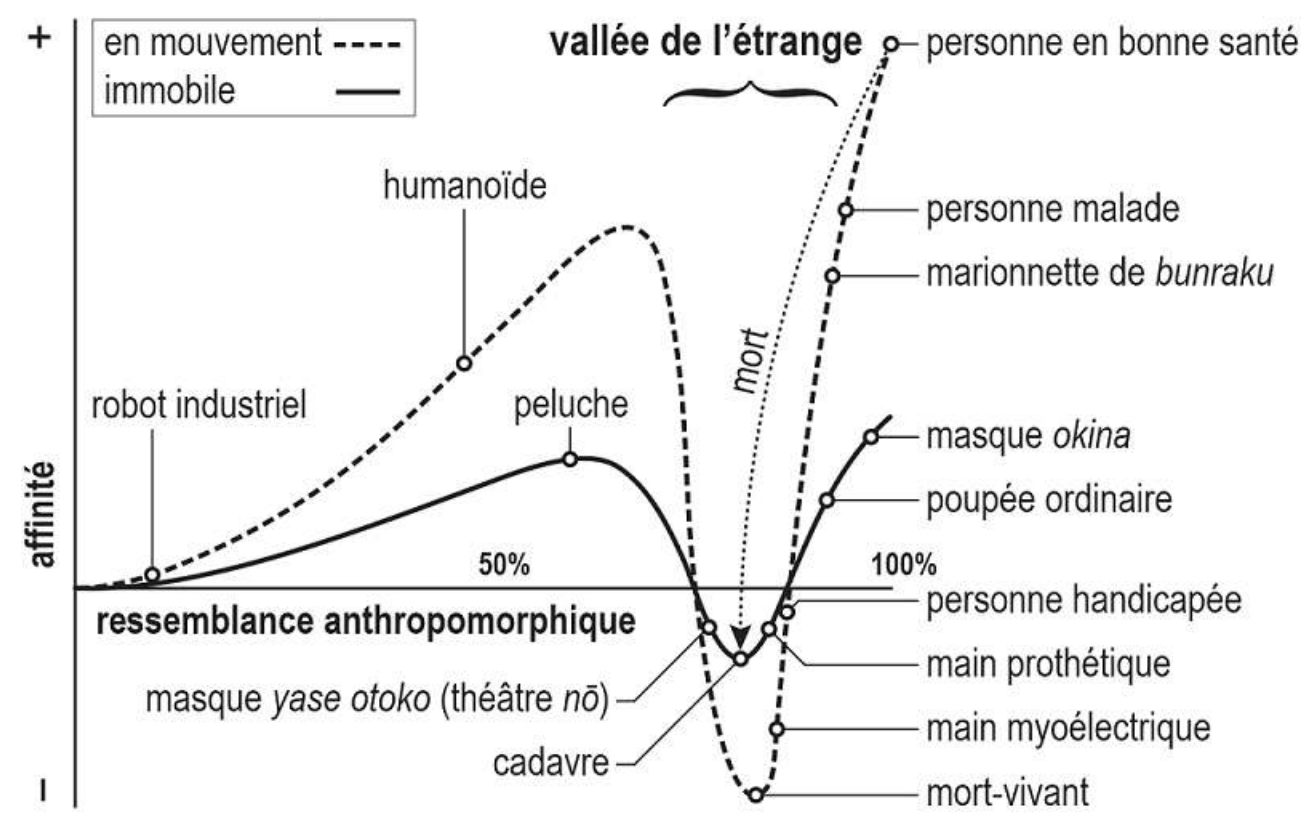

Quand on lui ajoute la dimension du mouvement, les pentes de la vallée de l'étrange deviennent plus abruptes. À sa mort, l'homme suit le trajet de la flèche en pointillé du graphique et sort de la courbe des objets en mouvement pour aller vers celle des objets inanimés

Karl F. MacDorman

\section{L'Effet du mouvement}

Pour les êtres humains et les autres animaux, le mouvement est synonyme de vie - il en est de même pour les robots. Sa présence modifie la forme de la vallée de l'étrange en amplifiant les pics et les creux, comme le montre la figure 2. Pour illustrer mon propos, prenons l'exemple d'un robot industriel. Lorsqu'il est éteint, c'est une simple machine couverte de graisse. Mais une fois qu'il est programmé pour bouger comme une main humaine, nous commençons à ressentir une certaine affinité pour lui (dans ce cas, la vitesse, l'accélération et la décélération du robot doivent ressembler au mouvement humain). À l'inverse, si l'on ajoute le mouvement à une main prothétique déjà presque en bas de la vallée de l'étrange, notre sentiment de malaise vis-à-vis d'elle s'intensifie grandement.

Certain de nos lecteurs savent que la technologie récente a rendu possible l'extension et la contraction automatique des doigts des mains prothétiques. La figure 3 montre le meilleur modèle disponible sur le marché conçu par un fabricant à Vienne. Même en l'absence de l'avant-bras, le simple fait de penser à remuer les doigts produit un faible courant dans les muscles du bras, qui peut être détecté par un électromyogramme. Lorsque la prothèse détecte le courant via des électrodes placées à la surface de la peau, elle amplifie le signal afin de mettre en marche un petit moteur qui remue les doigts. Le seul fait que cette prothèse myoélectrique soit capable de bouger a de grande chance d'être révulsant pour une personne parfaitement valide. Si une personne avec une main prothétique motorisée serrait la main à une femme dans le noir, elle en hurlerait certainement de terreur! 
Figure 3 : Une main myoélectrique

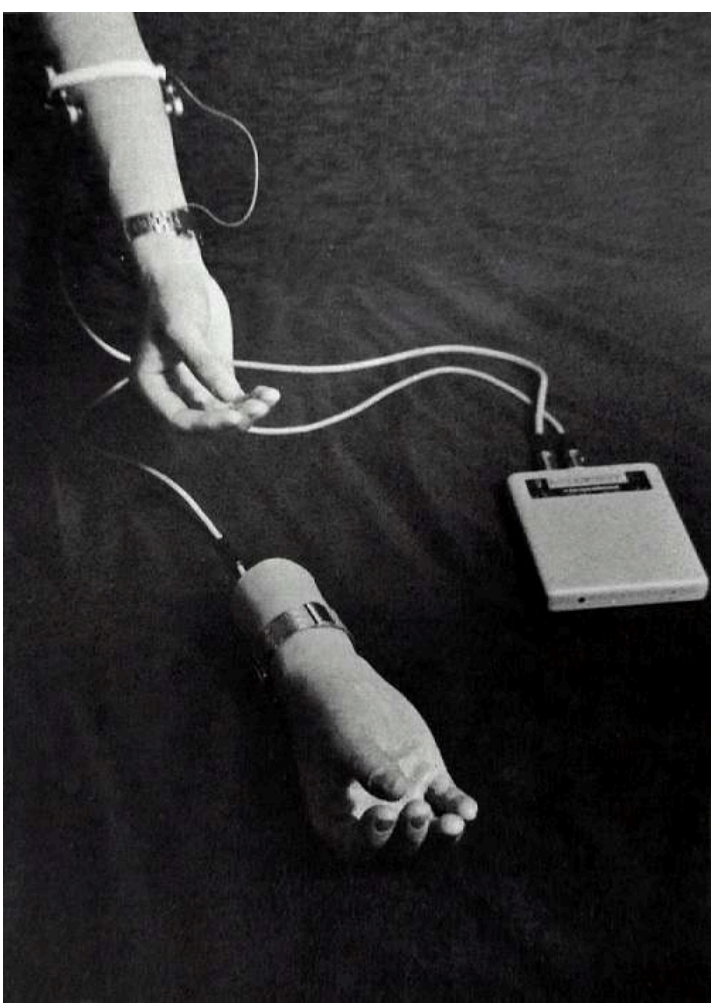

Un bras humain manipule une main myoélectrique qui s'appelle la « Main de Vienne ».

Masahiro Mori

Puisque les effets négatifs du mouvement sont évidents, même avec une main prothétique, un robot complet mettrait en exergue ce sentiment d'inquiétude. Dès lors, que penser si nous étions entourés de nombreux robots? Imaginez cette situation : un artisan se réveille dans la nuit et se rend à son atelier rempli de mannequins afin d'y rechercher quelque chose, et ces mannequins se mettent à bouger... Une vraie histoire d'horreur!

Les effets liés au mouvement ont été observés à l'exposition universelle de 1970 à Osaka, au Japon. Les programmes pour l'événement évoquaient la construction de robots extrêmement sophistiqués. Par exemple, un robot disposait de 29 paires de muscles artificiels dans le visage (encore plus qu'un être humain) afin qu'il puisse sourire de façon humaine. Selon son concepteur, le sourire est le produit d'une séquence de déformations du visage, la vitesse de ces déformations étant un paramètre crucial. Lorsque cette vitesse est réduite de moitié pour essayer de faire sourire le robot plus lentement, l'expression du bonheur devient inquiétante et vire au sinistre. Ainsi, en fonction du mouvement et de ses variations, quelque chose de presque humain comme un robot, une marionnette ou une main prothétique - peut aisément se retrouver dans la vallée de l'étrange.

\section{L'Évasion par le design}

Nous espérons concevoir et construire des robots et des mains prothétiques qui ne tombent pas dans la vallée de l'étrange. Néanmoins, en raison du risque inhérent qui existe pour essayer d'atteindre le second pic en visant la ressemblance parfaite, je 
recommande aux concepteurs de se fixer comme objectif le sommet du premier pic. L'anthropomorphisme y est suffisamment visible pour être associée à un fort sentiment d'affinité. Pour moi, il est même possible de créer un niveau d'affinité acceptable en poursuivant un concept non anthropomorphique, les concepteurs de robots devraient y réfléchir. Pour illustrer ce phénomène, considérons les lunettes de vue. Les lunettes, de par leur design, ne sont pas conçues pour ressembler à de vrais globes oculaires, mais contribuent pourtant à créer une nouvelle esthétique charmante pour les yeux de ceux qui les portent. Ce même principe devrait être suivi lors de la conception de mains prothétiques, afin qu'elles n'aient pas l'air pitoyable mais soient plutôt tendance.

Figure 4 : Une main en bois sculpté

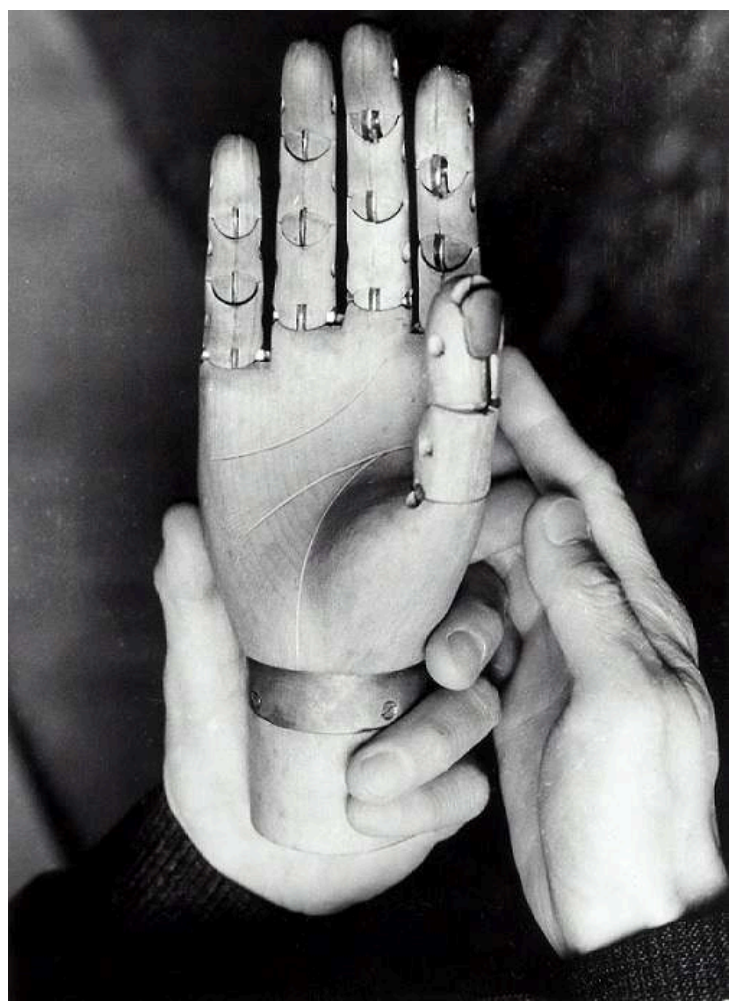

Un modèle d'une main fabriqué par un sculpteur sur bois de statue de Bouddha.

Masahiro Mori

Autre exemple : considérons le modèle d'une main humaine fabriqué par un sculpteur sur bois de statues de Bouddha (Figure 4). Les doigts se plient librement aux articulations. La main n'a pas d'empreintes et garde la couleur naturelle du bois, mais sa rondeur et ses belles courbes ne suscitent pas de sentiment inquiétant. Peut-être qu'une main de ce genre pourrait servir d'exemple pour la conception de robots.

\section{Une explication de l'inquiétante étrangeté}

En tant que personnes en bonne santé, nous nous trouvons au sommet du second pic de la figure 2 (en mouvement). Puis, à notre mort, nous ne pourrons logiquement plus bouger, notre corps se refroidira et notre visage deviendra livide. Ainsi, notre décès peut être considéré comme un déplacement du sommet du second pic jusqu'au fond de la vallée de l'étrange (inanimée), comme le parcours de la flèche en pointillé sur la 
figure 2 l'indique. Heureusement, cette flèche descend vers la vallée inanimée et non pas vers celle corrélée au mouvement. Si nous devions y tomber, nous rejoindrions la figure cauchemardesque du mort-vivant.

Je pense que cette descente élucide le profond mystère qu'entoure la vallée de l'étrange. Pourquoi sommes-nous envahis par ce sentiment de malaise ? En quoi nous est-il nécessaire? Je n'ai pas encore suffisamment réfléchi à ces questions, mais je pense que tout cela est intrinsèquement lié à notre instinct de survie ${ }^{5}$.

Via la recherche en robotique, nous devons commencer à établir une carte précise de la vallée de l'étrange qui nous permette de comprendre ce qui fait de nous des êtres humains. Cette carte est aussi nécessaire pour créer - sans forcément poursuivre un design anthropomorphique - des appareils auprès desquels les gens peuvent ressentir de l'affinité.

\section{Épilogue}

Mori Masahiro a préparé la déclaration suivante ${ }^{6}$, datée du 18 août 2005, afin qu'elle soit partagée pendant l'atelier de Discussions sur la Vallée de l'Étrange (Views of the Uncanny Valley), tenue entre le 5 et 7 décembre 2005 à la cinquième Conférence Internationale sur les Robots Humanoïdes à Tsukuba au Japon :

«Ce fut un grand honneur et un plaisir pour moi d'apprendre que l'atelier sur la vallée de l'étrange, concept que j'avais suggéré il y a trente-cinq ans, allait aborder ce thème dans des domaines aussi variés que les neurosciences, la reconnaissance des formes, l'intelligence artificielle, la psychologie et la sociologie. Malheureusement, je dois présider une autre réunion, ce qui m'empêche d'être présent aujourd'hui. Afin de remédier à mon absence, je ferai ici deux brefs commentaires sur le sujet :

Premièrement, le visage d'un défunt peut en effet être dérangeant : il perd sa couleur, sa vitalité, et ne cligne plus des yeux. Cependant d'après mon expérience, il peut parfois donner une impression d'apaisement, plus que ne pourrait le donner le visage d'une personne vivante. Les morts sont libérés des soucis de la vie quotidienne et je pense que c'est la raison pour laquelle leur visage paraît si calme et apaisé. Il y a toujours dans notre esprit ce conflit antinomique qui dit que ce que l'on obtient d'un côté, on le perd de l'autre. Un tel conflit se reflète sur notre visage par des expressions de malaise et d'angoisse. Mais lorsqu'une personne meurt, il ou elle se libère de cette opposition et l'expression de son visage devient calme. Si tel est le cas, où cette expression devraitelle être située sur la courbe de la vallée de l'étrange ? Cette question est l'une de mes préoccupations actuelles.

Deuxièmement, j'avais positionné autrefois les personnes en bonne santé au sommet de la courbe, du côté droit de la vallée de l'étrange. Toutefois, j'en suis arrivé à penser récemment qu'il existe quelque chose de plus séduisant et de plus agréable encore que les personnes vivantes, quelque chose à placer plus encore sur la droite : le visage d'une statue bouddhiste, en tant qu'expression artistique de l'idéal humain. On trouve ce genre de visages dans des exemples comme le Hōkan Miroku 宝冠弥勒 un des Miroku Bosatsu 弥勒菩薩 (Bodhisattva Maitreya) du temple Kōryū-ji 広隆寺 à Kyoto, ou encore à Nara, dans le Miroku Bosatsu du temple Chūgū-ji 中宮寺 [figure 5] et le Gakkō Bosatsu 月光菩薩 (Candraprabha) du temple Yakushi-ji 薬師寺. Ces visages sont pleins d'élégance, ne sont affectés par aucun souci de la vie et possèdent une aura de dignité. 
Je pense que ce sont ces choses-là qui doivent être positionnées au plus haut point de la courbe.

Bien que je sois moi-même à l'origine de la théorisation du concept de vallée de l'étrange, je n'en ai pas encore fait le tour. J'espère que les deux points que je viens de mentionner aideront à faire avancer les recherches à ce sujet. "

\section{BIBLIOGRAPHIE}

BURLEIGH Tyler J. et SCHOENHERR Jordan R., « A reappraisal of the uncanny valley: Categorical perception or frequency-based sensitization? », Frontiers in Psychology, vol.5, n¹488, 2014, p. 1-19, doi : $\underline{10.3389 / \text { fpsyg.2014.01488. }}$.

BUTLER Matthew et JOSCHKO Lucie, « Final Fantasy or The Incredibles: Ultra-realistic animation, aesthetic engagement and the uncanny valley ", Animation Studies, vol.4, 2009, p.55-63.

FISKE, Susan T., CUDDY, Amy J. C. et GLICK, Peter, « Universal dimensions of social cognition: Warmth and competence ", Trends in Cognitive Sciences, vol.11, n², 2007, p.77-83, doi : $\underline{10.1016 /}$ j.tics.2006.11.005.

FREEDMAN Yacov, «Is it real... or is it motion capture? The battle to redefine animation in the age of digital performance », The Velvet Light Trap, vol.69, 2012, p.38-49, doi : $\underline{10.1353 / v l t .}$ $\underline{2012.0001 .}$.

FREUD Sigmund, «Das Unheimliche » (L'inquiétante étrangeté). Imago. Zeitschrift für Anwendung der Psychoanalyse auf die Geisteswissenschaften, vol.5, 1919, p.297-324 doi : $\underline{\text { 10.11588/diglit.25679.17. }}$. GRAY Kurt et WEGNER Daniel M., « Feeling robots and human zombies: Mind perception and the uncanny valley », Cognition, vol.125, n¹, 2012, p.125-130, doi : 10.1016/j.cognition.2012.06.007.

JENTSCH Ernst, «Zur Psychologie des Unheimlichen » (Sur la psychologie de l'inquiétante étrangeté), Psychiatrisch-neurologische Wochenschrift, vol.8, 1906, p.195-198, 203-205.

JOCHUM Elizabeth et GOLDBERG Ken, « Cultivating the uncanny: The Telegarden and other oddities ", dans HERATH, Damith, KROOS Christian et Stelarc (dir.), Robots and art: Exploring an unlikely symbiosis, Singapore, Springer, 2015, p.149-175.

KAGEKI Norri, « An uncanny mind », IEEE Robotics and Automation, vol.19, n², 2012, p.112-108, doi : 10.1109/MRA.2012.2192819.

KÄTSYRI Jari, FÖRGER Klaus, MÄKÄRÄINEN Meeri et TAKALA Tapio, « A review of empirical evidence on different uncanny valley hypotheses: Support for perceptual mismatch as one road to the valley of eeriness ", Frontiers in Psychology, vol.6, n³90, 2015, p.1-16, doi : 10.3389/fpsyg. $\underline{2015.00390 .}$.

MACDORMAN Karl F., « Androids as experimental apparatus: Why is there an uncanny valley and can we exploit it? ", dans CogSci-2005 Workshop: Toward Social Mechanisms of Android Science, Stresa, Italy, July 25-26, 2005, p.108-118. 
MACDORMAN, Karl F., « In the uncanny valley, transportation predicts narrative enjoyment more than empathy, but only for the tragic hero ", Computers in Human Behavior, vol.94, 2019, p. 140-153 doi : 10.1016/j.chb.2019.01.011.

MACDORMAN Karl F. et CHATTOPADHYAY Debaleena, « Reducing consistency in human realism increases the uncanny valley effect; increasing category uncertainty does not ", Cognition, vol.146, 2016, p.190-205 doi : 10.1016/j.cognition.2015.09.019.

MACDORMAN Karl F. et ENTEZARI Steven, « Individual differences predict sensitivity to the uncanny valley », Interaction Studies, vol.16, n², 2015, p.141-172, doi : 10.1075/is.16.2.01mac.

MACDORMAN Karl F. et ISHIGURO Hiroshi, « The uncanny advantage of using androids in social and cognitive science research ", Interaction Studies, vol.7, n³, 2006, p.297-337 doi : $\underline{10.1075 / \text { is. }}$. 7.3.03mac.

MACDORMAN Karl F., VASUDEVAN Sandosh K. et HO Chin-Chang, « Does Japan really have robot mania? Comparing attitudes by implicit and explicit measures », AI \& Society, vol.23, n4, 2009, p. -485-510 doi :10.1007/s00146-008-0181-2.

MATHUR Maya B. et REICHLING David B., « Navigating a social world with robot partners: A quantitative cartography of the uncanny valley ", Cognition, vol.146, 2016, p.22-32, doi : $\underline{10.1016 /}$ j.cognition.2015.09.008.

MITCHELL Wade J., SZERSZEN Sr. Kevin A., LU Amy S., SCHERMERHORN Paul W., SCHEUTZ Matthias et MACDORMAN Karl F., « A mismatch in the human realism of face and voice produces an uncanny valley », i-Perception, vol.2, n¹, 2011, p.10-12, doi : 10.1068/i0415.

MORI Masahiro, « Bukimi no tani genshō » (Le phénomène de la vallée de l'étrange), Energy, vol.7, $\mathrm{n}^{\circ} 4,1970$, p.33-35.

MORI Masahiro, «La vallée de l'étrange » (trad. d'YAYA Isabel), Gradhiva, vol.15, 2012b, p.26-33, doi : $10.4000 /$ gradhiva.2311.

MORI Masahiro, « The uncanny valley » (trad. de MACDORMAN Karl F. et KAGEKI Norri), IEEE Robotics and Automation, vol.19, n², 2012a, p.98-100, doi : 10.1109/MRA.2012.2192811.

REICHARDT Jasia, « Human reactions to imitation humans, or Masahiro Mori's Uncanny Valley » dans Robots: Fact, Fiction, and Prediction (1st ed.), New York, Viking, 1978a, p. 26-27

REICHARDT Jasia, Les robots arrivent (trad. de COHEN André-Charles), Paris, Éditions du Chêne, 1978.

SAYGIN Ayse P., CHAMINADE Thierry, ISHIGURO Hiroshi, DRIVER Jon et FRITH Chris, « The thing that should not be: Predictive coding and the uncanny valley in perceiving human and humanoid robot actions ", Social Cognitive and Affective Neuroscience, vol.7, n 4, 2012, p.413-422, doi : $10.1093 /$ scan/nsr025.

SEYAMA Jun'ichiro et NAGAYAMA Ruth S., "The uncanny valley: The effect of realism on the impression of artificial human faces ", Presence: Teleoperators and Virtual Environments, vol.16, $\mathrm{n}^{\circ} 4$, 2007, p.337-351, doi : $\underline{10.1162 / \text { pres.16.4.337 }}$

TINWELL Angela, GRIMSHAW Mark, ABDEL NABI Debbie et WILLIAMS Andrew, « Facial expression of emotion and perception of the uncanny valley in virtual characters ", Computers in Human Behavior, vol.27, n², 2011, p.741-749, doi : 10.1016/j.chb.2010.10.018.

TONDU Bertrand et BARDOU Nicole, « A new interpretation of Mori's uncanny valley for future humanoid robots », International Journal of Robotics and Automation, vol.26, n³, 2011, p.337-348, doi : $\underline{10.2316 / J o u r n a l .206 .2011 .3 .206-3348 ~}$ 
URGEN Burcu A., KUTAS Marta et SAYGIN Ayse P., « Uncanny valley as a window into predictive processing in the social brain », Neuropsychologia, vol.114, 2018, p.181-185, doi : 10.1016/ j.neuropsychologia.2018.04.027.

YAMAMOTO Shichihei, « Naze nihonjin niwa robotto arerugī ga nai no ka? » (Pourquoi les Japonais ne sont-ils pas allergiques aux robots? Why aren't Japanese allergic to robots?), Gendai No Espuri, vol.187, 1983, p.136-143.

\section{NOTES}

1. Mori Masahiro (12 février 1927-) est professeur émérite de l'université de technologie de Tokyo (Tōkyō Kōgyō Daigaku 東京工業大学), dans laquelle il a dirigé la recherche en robotique pendant près de deux décennies. Il est également président de l'Institut Mukta (Jizai kenkyūjo 自 在研究所) et président honoraire de la Société de robotique du Japon (Nihon robottogakkai 日本 ロボット学会). Il a terminé son doctorat en ingénierie à l'Université de Tokyo et a servi dans leur faculté entre autres. Mori est célèbre pour avoir introduit le concept de la vallée de l'étrange en 1970, qui a bénéficié d'une grande audience dans des domaines aussi variés que la robotique humanoïde et l'animation par ordinateur, jusqu'à la psychologie perceptuelle et les neurosciences cognitives. Il est connu pour ses réflexions mêlant robotique et bouddhisme, détaillées notamment dans Le Bouddha dans le robot, ainsi que dans de nombreux autres ouvrages. Mori a grandement contribué à la recherche et à l'enseignement en robotique et en automatisation, fondant certains des premiers concours de robotique. Il a reçu de nombreux prix de sociétés savantes, le Prix culturel de la radiodiffusion de la NHK (Hōsō bunka-shō放送文化賞) en 1992. Il est également le récipiendaire de la Médaille d'honneur (Shijuhōshō褒章紫綬褒章) reçue en 1994 et de l'Ordre du soleil levant (Kunsantō kyokujitsu-chūjushō 重三等旭日中綬章) en 1999.

2. Organisation pour le Développement des Énergies Nouvelles et des Technologies Industrielles www.nedo.go.jp

3. Les robots industriels sont toutefois bien plus proches des êtres humains en termes d'apparence que les machines générales, en particulier en ce qui concerne leurs bras.

4. Il existe un autre courant de pensée expliquant la fascination ressentie à l'égard des robots par le fait qu'ils soient conçus pour dépasser et améliorer les humains.

5. En outre, je pense que ce sentiment d'étrangeté correspond à un instinct de conservation non pas pour nous protéger du vent, de l'eau ou de toute autre chose, somme toute assez différente du corps humain, mais pour nous protéger des choses qui nous semblent au contraire bien trop similaires au corps humain, telles que les cadavres ou d'autres espèces apparentées.

6. 私が35年前に提唱した「不気味の谷」の概念に関するワークショップで、神経科学、パ ターン認識、人工知能 (AI) 、心理学、社会学など、さまざまな分野のトピックを取り上げ ることになったことを知り、大变光栄に思っています。あいにく私は、別の会合の議長を 務める必要があるので、今日そちらに出席することができませんが、欠席の埋め合わせと して、このテーマに関して2点手短に見解を述べます。1.亡くなった人の顔は、確かに気味 の悪いものかもしれません。顔色や生気を失い、瞬きもしません。しかしながら、私の経 験では、それは生きている人の顔よりも、より安らかな印象を与えることがあります。死 人は人生の困難から解放されています。それが彼らの顔がとても穏やかで安らかに見える 理由だと思います。私たちの心の中には常に、一方を得ることで他方を失うという、自己 矛盾（二律背反）が存在します。そのような葛藤は、苦悩や不快感の表現として私たちの 顔に現れます。でも、人は死ぬと、この自己矛盾から解放されて、穏やかな表情になりま す。そうであ机ば、この表情は、「不気味の谷」曲線のどこに位置づけられるべきなので しょうか。この問題は私の現在の関心事の一つです。2.かつて私は、健康人を「不気味の 
谷」曲線の右側の最高点に位置づけていました。しかし最近では、人よりももっと人の心 をひきつけ、好感が持てるものがあり、それが曲線のさらに右側に位置づけられると思う ようになりました。それは、人間の理想の芸術的表現とされる、仏像の顔です。そのよう な顔は、例えば、京都・広隆寺の弥勒菩薩（マイトレーヤ仏）の一つである宝冠弥勒や、 奈良・中宮寺の弥勒菩薩、奈良・薬師寺の月光菩薩（チャンドラプラバ）などに見られま す。これらの顔は、気品に満ち、生活の心配などはものともせず、独特の高潔な雾囲気を 漂わせています。こういった要素があるものこそが、まさに曲線の最高点に置かれるべき だと思います。「不気味の谷」の概念を自分が創案したにもかかわらず、私は未だにそれ を深く吟味していません。上記 2 点が、このトピックにおける研究の進歩に役立つことを 原っています。

\section{RÉSUMÉS}

Dans son essai précurseur publié en 1970, Masahiro Mori observe que l'affinité pour les robots augmente en fonction de leur ressemblance à l'humain, jusqu'à un certain point. Cependant, entre le premier pic d'affinité et le second d'indifférenciation humaine se trouve la vallée de l'étrange. Mori conseille aux créateurs d'éviter cette vallée et de viser le premier pic. Confrère dans la recherche en robotique, le traducteur de Mori réfléchit sur l'importance de l'essai et sur son influence durable en robotique et animation 3D au Japon et aux États-Unis. La vallée de l'étrange est vue positivement comme diagnostic afin d'améliorer les théories d'interaction humaine, dont les androïdes sont équipés. La recherche sur la vallée de l'étrange a explosé dans divers domaines depuis sa présentation en 2005 lors d'un atelier. Cet intérêt devrait s'intensifier puisque les chercheurs construisent des robots, des avatars et autres interfaces pour la technologie informatique de plus en plus ressemblant à l'humain.

Masahiro Mori observed in his seminal essay, published in 1970, that affinity for robots increases with their human similarity up to a moderate degree. However, between this first peak in affinity and the second, higher peak of human indistinguishability lies an uncanny valley. Mori advises designers to avoid this valley by aiming solely for the first peak. As a fellow robotics researcher, Mori's translator reflects on the significance of the essay and its sustained influence on robotics and 3D animation in Japan and the United States. The uncanny valley is viewed positively as a diagnostic for improving theories of human interaction as implemented in androids. The investigation of the uncanny valley has exploded since a workshop in 2005 in various scientific fields. This interest is expected to intensify as researchers build ever more humanlike robots, avatars, and other kinds of interfaces for computing technology.

\section{INDEX}

Mots-clés : histoire des techniques, anthropomorphisme, interaction homme-machine, robotique humanoïde, imaginaire

Keywords : history of technology, anthropomorphism, human-robot interaction, robot, humanoid robotics, android science

Thèmes : Entretiens et leçons 


\section{AUTEURS}

\section{KARL F. MACDORMAN}

Karl F. MacDorman est Doyen Associé en Affaires Académiques à la faculté d'Informatique de l'Université de l'Indiana. Il fut précédemment Maitre de Conférences à l'École d'Ingénieurs de L'Université d'Osaka. Il a reçu son doctorat en Sciences de l'Informatique à l'Université de Cambridge en 1997 et sa licence en Sciences de l'Informatique à l'Université de Californie à Berkeley en 1988. Il a publié plus de 100 articles sur l'interaction homme-robot, l'apprentissage automatique et les sciences cognitives. Les principaux centres d'intérêt de ses recherches comprennent l'émergence et le fondement des symboles, le développement de la robotique cognitive et la science androïde. Sa traduction en anglais de La Vallée de l'étrange a fait date. 